\title{
GOOD LABORATORY PRACTICE
}

\author{
Ashok Chidanand Alatgi ${ }^{1}$, Sanjeev Bhaurao Chougule ${ }^{2}$
}

1 Professor of Pathology, Department of Pathology, J. N. Medical College, Nehru Nagar, Belagavi, Karnataka, India.

${ }^{2}$ Senior Lecturer, Department of Hospital Administration, J. N. Medical College, Nehru Nagar, Belagavi, Karnataka, India.

\section{ABSTRACT}

In the present era of rapidly evolving technology and evidence based medicine, Good Laboratory Practice (GLP) shall play an important role in ensuring, consistency, reliability, reproducibility and quality of laboratory tests. It provides a frame work for laboratories to plan, perform, monitor, record and report their activities. It also reflects the quality of laboratory services during any assessments and accreditation process. The quality assessment statement is not GLP of compliance statement. The poor laboratory practice found were. ${ }^{1}$

1. Equipment has not been calibrated to standard form, therefore giving wrong results.

2. Incorrect and inaccurate accounts of the actual laboratory studies.

3. Inadequate test system.

\section{KEYWORDS}

Technicians, Laboratory, GLP, Haematology, Histopathology, Cytology, Microbiology, Bio-Chemistry, Bio-Hazards.

HOW TO CITE THIS ARTICLE: Alatgi AC, Chougule SB. "Good laboratory practice." Journal of Evolution of Medical and Dental Sciences 2015; Vol. 4, Issue 103, December 24; Page: 16901-16906, DOI: 10.14260/jemds/2015/2543

\section{INTRODUCTION}

Good Clinical Practice (GCP) is an international ethical and scientific quality standard for designing, conducting, recording and reporting trials that involve the participation of human subjects. Compliance with this standard provides public assurance that the rights, safety and well-being of trial subjects are protected consists with the principles that have their origin in the declaration of Helsinki (ICH GCP guideline). ${ }^{2}$

Good Laboratory Practice (GCP) is intended to promote the quality and validity of test data. It is a managerial concept covering the organizational process and the conditions under which laboratory studies are planned, performed, monitored, recorded and reported (OECD GLP guideline). ${ }^{2}$

\section{HISTORY}

Good Laboratory Practice (GLP) was first introduced in New Zealand and Denmark in 1972 .

In the early 1970s FDA became aware of cases of poor laboratory practice all over the United States. One of the laboratory that went under such investigation was Industrial Bio Test. It was run by Procter and Gamble. It was discovered that mice that they have used to test for cosmetics such as lotion and deodorants had developed cancer and died. The laboratory threw the mice and covered the results.

A few years later by the Organization for Economic CoOperation and Development (OECD) helped promulgate GLP to many countries. GLP, a data quality system, should not be confused with standards of laboratory safety - appropriate gloves, glasses and clothing to handle lab material safely. ${ }^{1}$

\section{DEFINITION}

Good Laboratory Practice (GLP) embodies a set of principles that provides a framework within which laboratory studies are planned, performed, monitored, recorded, reported and archived.

Financial or Other, Competing Interest: None.

Submission 23-01-2015, Peer Review 29-01-2015,

Acceptance 18-12-2015, Published 24-12-2015.

Corresponding Author:

Dr. Ashok Chidanand Alatgi,

Department of Pathology,

J. N. Medical College,

Nehru Nagar, Belagavi,

Karnataka, India.

E-mail: aalatgi@gmail.com

DOI:10.14260/jemds/2015/2543
These studies are undertaken to generate data by which the hazards and risks to users, consumers and third parties including the environment can be assessed for pharmaceuticals (only pre-clinical studies), agrochemical, cosmetics, food, additives, food additives and contaminant, novel foods, bio-seeds, detergents, etc. GLP helps assure regulatory authorities that the data submitted are a true reflection of the results obtained during the study and can therefore be relied upon when making risk/safety assessments. ${ }^{2}$

\section{Objectives of GLP}

- GLP makes sure that the data submitted are a true reflection of the results that are obtained during the study.

- GLP also makes sure that data is traceable.

- Promotes international acceptance of tests.

\section{Mission of GLP}

- $\quad$ Test systems.

- Archiving of records and materials.

- Apparatus, material and reagent facilities.

- Quality assurance programs.

- Performance of the study.

- Reporting of study results.

- Standard Operating Procedures (SOP).

- Personnel and test facility organization Standard Operating Procedures (SOP).

- Written procedures for laboratories program.

- They define how to carry out protocol specified activities.

- Most often written in a chronological listing of action steps.

- They are written to explain how the procedures are suppose to work.

\section{Standard Operative Procedure (SOP)}

- Routine inspection, cleaning, maintenance, testing and calibration.

- Action to be taken in response to equipment failure.

- Analytical methods.

- Definition of raw data.

- $\quad$ Keeping records, reporting, storage, mixing and retrieval of data. 
Properly designed SOPs will bring the following benefits to the laboratory:

- Standardized consistent procedures minimizes personto-person and test-to-test variability.

- Demonstration of management commitment to quality as part of the SOP approval process.

- $\quad$ Ease of documenting complicated techniques in study protocols and reports (A simple reference to the procedure should often suffice).

- Continuity in case of personnel turnover.

- A means of communication in case of audit visits, technology transfer. etc.

\section{Facilities-Buildings}

Test facilities should have suitable size construction and location to meet the requirement of the study and to minimize disturbance that could interfere with the study. They should be designed to provide an adequate degree of separation of the diverse elements of the study.

Separation ensures that disturbances are minimized and that different activities do not interfere with one another or adversely affect the study.

\section{Regents and Material. ${ }^{2}$}

The reagent used are specified in the standard operating procedures.

Purchasing and testing should be handled by quality assurance program.

Reagents and solution shall be labeled, detoriated elated or out dated reagents and solution shall not be used. Include the date of opening of reagents, storage of reagents at ambient temperature the reagent expiry date should be noted before using.

\section{Instrumentation - Validation}

- This is a process necessary for any analytical laboratory.

- Data produced by "faulty" instruments may give the appearance of valid data.

- $\quad$ The frequency for calibration, re-validation and testing depends on the instruments and extent of its use in the laboratory.

\section{SAMPLE TRACKING}

Must maintain the unmistakable connection between set of analytical data and the specimen or samples from where they obtained. The original source of specimen or samples must be recorded and connected with set of analytical data. The sample or specimen should be adequately mixed or added the preservative used. This should be informed to analyst.

\section{ANALYST CERTIFICATION}

An acceptable proof of satisfactory training and competence with specific laboratory procedures must be established for each analyst. The qualification can come from education, experience or additional training, but it should be documented. The staff should be adequate.

A review of all job descriptions, annually or in the event of any reorganization helps the facility management to ensure that their organization is coherent.

\section{REPORTING RESULTS}

There are two basic types of report that might be produced when reporting results from analytical work.

1. Analytical report; a formal report which may be issued on completion of work detailed in analytical plan.

2. Analytic results: Documents containing just the results, which is usually issued rapidly on completion of sample analysis on a given day.

\section{Documentation and Maintenance of Records}

Maintenance of all records provide documentation, which may be required in the event of legal challengers due to repercussions of decisions, based on original analytical results. General guidelines followed in regulated laboratories is to maintain records for at least five years. The length of time over which laboratory records should be maintained will vary with the situation.

The analytical report should have sign and date by analytical project manager to indicate acceptance of responsibility for the validity of the data reported. The extent of compliance with these principles should be indicated.

\section{Documentation and Maintenance of Records}

- Maintenance of all records provide documentation, which may be required in the event of legal challenges due to repercussions of decisions based on the original analytical results.

- General guidelines followed in regulated laboratories is to maintain records for at least five years.

- Length of time over which laboratory records should be maintained will vary with the situation. ${ }^{2}$

\section{Haematology Quality Assessmemt. ${ }^{3}$}

Laboratories must have an established quality assessment program as mandated by subpart K-Quality systems for nonwired testing of the clinical laboratory improvement amendments of 1988 (CLIA 88).

A common approach to the development of a quality assessment program is to divide it into three components: (1) Pre-examination which deals with all aspects affecting the test outcome occurring prior to the testing procedure. (2) Examination which incorporates all aspects affecting the testing procedure itself and (3) Post examination which deals with aspects affecting the test outcome occurring after the testing procedures.

\section{Pre-Examination Component}

The testing process begins with the test order. Patient test requisitions should be designed to be user friendly and to provide adequate patient information. At a minimum this information should include the Patient's Name or Unique Patient Identifier, Age, Sex, Diagnosis, Test to be Performed, and Source of the Sample. The patient should receive appropriate information to prepare for the test such as being informed to abstain from aspirin or aspiring like medication prior to platelets function testing. 
Sample collection Tubes, their Anticoagulants, and Typical Laboratory uses

\begin{tabular}{|c|c|c|}
\hline $\begin{array}{l}\text { Color } \\
\text { Code } \\
\text { Red } \\
\text { Light blue } \\
\text { Mottled } \\
\text { Red } \\
\text { Green } \\
\text { Purple or } \\
\text { Lavender } \\
\text { Pink } \\
\text { Gray }\end{array}$ & $\begin{array}{c}\text { Anticoagulant } \\
\text { No anticoagulant } \\
\text { Sodium citrate } \\
\text { No anticoagulant, but contains clot } \\
\text { activator and gel to facilitate separation of } \\
\text { serum from cells } \\
\text { Heparin } \\
\text { EDTA, K3 (liquid), or K2(spray-coated) } \\
\text { EDTA, K2 (spray-coated) } \\
\text { Potassium oxalate and sodium flouride }\end{array}$ & $\begin{array}{c}\text { Typical Laboratory Procedure } \\
\text { Antibody detection } \\
\text { Cholesterol } \\
\text { Osmotic fragility } \\
\text { CBC (K2 EDTA can also be used for routine blood bank } \\
\text { procedures; see below) } \\
\text { Blood bank procedures and whole blood hematology } \\
\text { procedures (different colored stopper for easy destination of } \\
\text { blood bank vs. hematology sample) } \\
\text { Glucose }\end{array}$ \\
\hline
\end{tabular}

$\mathrm{Pt}=$ Prothrombin time

APTT = Activated Partial Thromboplastin Time

\section{Venipuncture}

The venipuncture phlebotomy technique removes blood from a vein for laboratory testing using a sterile tube system.

The vein is selected by placing the tourniquet on the patient's upper arm and inspecting the forearm for a prominent vein. The median cubital vein is typically used, but the cephalic vein is also appropriate.

\section{Capillary Puncture}

The capillary puncture technique allows a small, but adequate amount of blood to be obtained for laboratory testing. This is especially important in the pediatric patient (i.e. infant) who has a lower total blood volume.

\section{Phlebotomy Safety}

These standards promote safety through education, good work practice, use of Personal Protective Equipment (PPE), and use of medical devices that minimize exposure risk. Each laboratory should develop an exposure control plan to address these issues.

\section{Tourniquet}

Various tourniquets including rubber straps blood pressure cuffs, and rubber tubing are available. Placement of the tourniquet on the patient's upper arm increases the resistance in venous blood flow resulting in the distension of veins below the tourniquet. The phlebotomist is then able to visualize and/or palpate the veins to identify the "good" vein.

\section{Lancets}

Various sterile disposable lancets are available for performing capillary puncture. The design of these devices has been improved to minimize the discomfort and minimize the quality of the sample.

\section{Bright-Field Microscopy}

The magnifying system of the compound microscope using two sets of lenses to form an enlarged image. The first lense system is the objective, which projects the primary image plane to a location approximately from the top of the microscope's body the distance of the back focal plane of the objective to the eye piece is termed the optical tube length $(110 \mathrm{~mm})$. The second lens system is the eye piece located above the primary image plane. The total magnification is the product of the magnification of the first and second lens systems (Objective magnification time's eyepiece magnification).

\section{Automated Blood Cell Counting Instruments}

The evaluation of instrumentation in hematology began in the mid 1950s. With the advent of the first single automated blood cell counter, manual hemacytometer blood cells counts for erythrocyte enumeration were replaced. In general, automated blood cell counters provide data with increased reliability, precision and accuracy. The impedance principle of blood cell counting is based on the increased resistance that occurs when a blood cell with poor conductivity passed through an electrical field. The number of pulses indicate the blood cell count, and the amplitude (i.e. height) of each pulse is proportional to the volume of the cell. The optical lightscattering principle of blood cell counting is based on light scattering measurements obtained as a single blood cell pass through a beam of light (optical or laser). Blood cell create forward scatter and side scatter is a measurement of cell size and the degree of side scatter is a measurement of cell complexity or granularity.

These automated determine additional reticulocyte parameters including reticulocyte hemoglobin concentration (CHr or Ret $\mathrm{He}$ ) reticulocyte Mean Cell Volume (MCVr) and Immature Reticulocyte Fraction (IRF). The reticulocyte hemoglobin concentration is determined by directly measuring the reticulocyte's hemoglobin by light scattering characteristics. The presence of increased number of immature reticulocytes can be used as an indicator of an erythropoietic response.

\section{Peripheral Blood Smear Staining}

For more than 100 years Ramanowsky stains have been used in the morphology classification of hematopoietic cells. Well stained blood smear is extremely important in the hematology laboratory because a wealth of information can be obtained from the blood smear. ${ }^{3}$

\section{Histopathology.4}

The space should be well ventilated fitted with exhaust fan. Approximate area may be 1,000-1,500 Sq.ft. For sample receiving is the basic which verifies Name, Age, Sex, IP No., Referral Consultant/Surgeon with Clinical Details and Diagnosis.

The laboratory gives one number - ref number. Some may start to from number of year followed by specimen. Eg2014 year first specimen numbers as HPR No. 140001.

\section{Check whether the specimen is sent in $10 \%$ Formalin or not}

Grossing is done according to specimen size and shape. It includes peripheral, tumor mass, surgical margin and respective lymph nodes when required. The bits are put in capsule, which is a metallic rectangular or circular with uniform pores in the wall. The capsule has got a locking system, so that it is well protected with tissue along with identifiable number. The number is written with lead pencil 
only. These capsules also fixed in and $10 \%$ formalin overnight. Next day morning they are hydrated and put into "Histokinette" beaker. The Histo-kinette is having twelve beakers of uniform size. The capsules are moved from one beaker to next beaker automatically. Six beakers for the various concentration of alcohol $-40 \%, 50 \%, 10 \%, 70 \%, 80 \%, 90 \%$, and $100 \%$ alcohol called as dehydration.

Clearing is done by xylene, chloroform, etc. Wax impregnation having a thermo-regulatory, the temperature varies from $50^{\prime} \mathrm{c}$ to $10^{\prime} \mathrm{c}$. This keeps wax in moulten or in liquid from.

\section{The scheduled for processing is as follows}

1. Fixation in $10 \%$ formaline $-1-10 \mathrm{Hrs}$.

2. Dehydration - 1-8 Hrs.

3. Clearing - 4-1 Hrs.

4. Wax impregnation $-1-8 \mathrm{Hrs}$

Block making with Leukherts "L" blocks. According to size of tissue 'L' blocks are adjusted. The tissue identity is also put around the block.

After block marking, the block is put on block holder of microtom. By heating block to 10 'c on slide warmer. The block is pressed on block holder and put in water. So that the wax becomes solid and intern holds block firmly. The block holder along with block is put on the microtome carrier. The microtome is the mechanical device, which cuts the slides at required thickness. The block is parallel to the cutting edge of the knife. First block is trimmed at $7-10 \mathrm{~mm}$ thickness. Then thickness is adjusted to $4-5 \mathrm{~mm}$. Ice is applied to the block. The serial sections are cut and taken on slide.

The slide along with section is kept in incubator for 10$15 \mathrm{~mm}$, so that the water can be removed and the section is well fixed to the slide. It is followed by haematoxylin and eosin staining procedure. Mount under DPX with appropriate size of cover slip. The reagent and chemicals used should be of reputed company. The commercially prepared hematoxyllin fades by 2-3 years.

The pathologist reports the slides. The report should be ready with following Name of Patient, Age/Sex, IP No./OPD No., Permanent Address, Complaints, Clinical Diagnosis, Microscopy, Impression.

The slides and reports are preserved for a period of 5-1 years. MLC cases slides can be kept for about 10 years. There are now automatic machines, which can replace the manual method of staining. ${ }^{4}$

\section{Cytology. ${ }^{5}$}

Cytology deals with three types of fluids: (1) Fluid cytology, (2) Gynaec cytology and (3) Fine Needle Aspiration Cytology (FNAC).

Patient should be well informed about the procedure to be performed. If the patient is female, a female attender/staff should be accompanied with patient. Room should be well ventilated. Ancillary investigation like x-ray, sonography and MRI scan should be accompanied.

The requisition form duly filled with Name, Age, Sex Inpatient/Outpatient Number and Permanent Address. Relevant history of the patient along with clinical diagnosis to be informed. Source and specific site (Left and right quadrant, etc.) nature of lesion-solid/cystic, functional, non-functional, etc.

Staff - Laboratory technician should be well trained in cytology procedures. He/she should be a certificate holder from a reputed university.

Fixation - Ether-alcohol or air dry for PAP and MGG stain respectively.
Procedures - The sample received at cytology section should be well identified by noting Name, Age/Sex, Inpatient No. or Outpatient No. along with history and clinical diagnosis. The staining procedures are Papanecolou (PAP stain) or May Granold - Giemsa (MGG) stain. The conventional PAP stain is better than rapid PAP stain. Nucleus and cytoplasmic morphology is clearer in PAP than MGG staining procedure.

2) Gynaec cytology- The test request form should include following - patient Name, Age, Sex, Inpatient No./Outpatient No., Date of specimen collection - Source of material submitted - cervical, endocervical, vaginal or other gynaecologic or nongynaecologic sites. Patient preparation - patient abstains from sexual intercourse for $48 \mathrm{hrs}$ prior to examination. Patient abstain from using vaginal medications, vaginal contraceptives or douches. The optimal time for PAP test is mid-cycle. Menses may interfere with test interpretation.

3) Details about gynaec specimens - Last menstrual period, pertinent clinical information - Routine examination, pregnancy, postpartum, hormone therapy, oral contraceptives, post-hysterectomy, post menopausal or pelvic irradiation.

The vaginal smear should be leveled the site from where the smear is obtained. The smear are fixed in ether-alcohol for 30 mins followed by PAP staining procedure.

\section{Fine Needle Aspiration Cytology (FNAC)}

Fine Needle Aspiration Cytology (FNAC) has revolutionized the diagnostic modalities.

Advantages - It is cheaper than any other surgical procedure. It can be done on OPD basis. It requires minimal instruments.

Disadvantages - 1) Uncooperative patient. 2) Bleeding diathesis may lead to complications like hematoma formation.

If the swelling is not accessible during routine procedures, it can be done by Ultrasound guided FNAC. Patient should be well explained and the written consent to be taken before procedure.

The requisition form should contain Patient's Name, Age/Sex, Inpatient/Outpatient Number, Permanent Address along with Phone Number. Relevant clinical history should be given along with clinical diagnostics. Local examination of the tumor to be furnished. Any relevant investigations can be given.

Once FNAC has been done, slides were prepared by putting one drop of aspirate on a clean, dry and dust free microscopic slide with the help of another slide and the smear were prepared. The slides with smear are fixed in either alcohol and fixed slides are used for Papanicolaou staining procedures and air dried smear are used for May-GrunwaldGiemsa staining procedures. The slides are mounted in DPX. The cytotechnician should always screen for whether material is there or not and then mount. The slides thus prepared are preserved for $3-5$ years. ${ }^{5}$

\section{MICROBIOLOGY. ${ }^{6}$}

There is a certain element of risk in anything you do, but the potential risks in a microbiology course are greater. Person who work in a Microbiology Lab may handle infectious agents in additional to other hazards, such as chemicals and radioactive materials. There have been many documented cases of lab personnel acquiring diseases due to their work. The microbiology laboratory is a unique environment that requires special practices and containment facilities in order to properly protect persons working with micro-organisms. Safety in the laboratory is the primary concern. The three main elements of safe containment of micro-organisms are (1) Good laboratory practices and technique, (2) Safety equipment and (3) Facility design. 


\section{Biosafety Levels and Practices}

The Center for Disease Control (CDC) and the National Institutes (NIH) have developed standard procedures providing protection against biological hazards. The publication, bio-safety in microbiological and biomedical laboratories.

(http://www.cdc.gov/OD/ohs/biosfty/bmbl4/bmbl4toc.htm ), provides specific description of microbiological practices, laboratory facilities and safety equipment and recommends their use in four Biosafety Level (BSLs).

\section{Bio-Safety Level 1(BSL1)}

Examples of BSL1 Agents: Bacillus subtilus, Naegleria gruberi, many Escherichia coli, Infectious canine Hepatitis Virus.

BSL1 containment is suitable for work involving wellcharacterized agents not known to cause disease in healthy adult humans and of minimal potential hazard to laboratory personnel and the environment. A BSL1 lab requires no special design features beyond those suitable for a well-designed and functional laboratory. Biological Safety Cabinets (BSCs) are not required. Work may be done on an open bench top and containment is achieved through the use of practices normally employed in a basic microbiological laboratory.

\section{Biosafety Level 2 (BSL2)}

Examples of BSL 2 Agents: Bacillus anthracis, Bordetella pertussis, Brucella SPP., Cryptococcus neoformans, clostridium botulinum, Clostridium tetani, Helicobacter pylori, most Salmonella spp., Yersinia pestis, Mycobacterium leprae, Shigella spp., Human Immunodeficiency Virus, Human blood.

The primary exposure hazards associated with organisms requiring BSL2 or through the ingestion inoculation and mucous membrane route. Agents requiring BSL to facilities are not generally transmitted by airborne routes, but care must be taken to avoid the generation of aerosols (Aerosols can settle on bench tops and become an ingestion hazard through contamination of the hands) or splashes. Primary containment devices such as BSCs and centrifuges with sealed rotors or safety cups are to be used as well appropriate personal protective equipments (e.g. gloves, laboratory coats, protective eyewear) as well environment contamination must be minimized by the use of hand washing sinks and decontamination facilities (Autoclave).

\section{Biosafety Level 3 (BSL3)}

Examples of BSL3 Agents: Mycobacterium tuberculosis, Salmonella type, Vesicular stomatitis virus, yellow fever virus, Francisella tularensis, Coxiella burnetii.

Laboratory personnel have specific training in handling these pathogenic and potentially lethal agents and are supervised by scientists who are experienced in working with these agents. These agents may be transmitted by the airborne route, often have a low infectious dose to produce effects and can cause serious or life-threatening disease. BSL3 emphasizes additional primary and secondary barriers to minimize the release of infectious organisms into the immediate laboratory and the environment. Additional features to prevent transmission of BSL3 organisms are appropriate respiratory protection, HEPA filtration of exhausted laboratory air and strictly controlled laboratory access.

\section{Biosafety Level 4 (BSL4)}

Examples of BSL4 Agents: smallpox virus, Ebola virus, hemorrhagic fever viruses.

This is the maximum containment available and is suitable for facilities manipulating agent that are dangerous/exotic agents, which post a risk of life-threatening disease. These agents have the potential for aerosol transmission, often have a low infection dose and produce very serious and often fatal disease; there is generally no treatment or vaccine available.

The level of containment represent an isolated unit, functionally and when necessary structurally independent of other areas. BSL4 emphasizes maximum containment of the infectious agents by complete sealing of the facility perimeter with confirmation by pressure decay testing; isolation of the researcher from the pathogen by his or her containment in a positive pressure suit or containment of the pathogens in a class III BSC line and decontamination of air and other effluents produced in the facility. ${ }^{6}$

\section{Biochemistry. ${ }^{7}$}

Biochemistry is an Interdisciplinary Science that integrates systematically the principles of mathematics, physics and chemistry to attempt to explain the distinctive characteristic of life processes in terms of structure function correlations. In recent years the fusion of biochemistry, cell biology and microbiology to form molecular biology has lead to spectacular advances in the understanding and control of biological processes in medicine, agriculture, pharmaceutics and the food and drink industry. Analysis means literally getting to the bottom of things, i.e. taking pieces.

Hazards arise from three main basic causes: from dangerous chemicals, from infected specimens sent for analysis and from faulty apparatus and instruments.

\section{HAZARDS FROM DANGEROUS CHEMICALS}

\section{Injury from chemicals results from:}

1. Direct contact:

a. With the skin, e.g. when pouring reagents or from breakage of containers.

b. With lips or mouth when pipetting, mouth pipetting should be forbidden.

c. With the esophagus and stomach if inadvertently swallowed.

2. Damage to the lungs from inhaling vapours or less likely fine powders.

3. Toxic effects of substances absorbed from the lungs, alimentary tract or skin on other tissues such as bone marrow, liver or kidney.

\section{Corrosive Chemicals}

Chemical burns of the skin can be caused by strong acids or alkalis, e.g. nitric, sulphuric and hydrochloric acid, sodium and potassium hydroxide and phenol (Solid or strong solutions). Take great care when opening bottles containing strong acids or ammonia, particularly when previously unopened or if the stopper sticks. This is best done in a fume cupboard.

\section{TOXIC CHEMICAL}

\section{Scheduled Poisons}

Such as cyanide and barbiturates are best kept locked in a cupboard or small store. Always measure reagents containing such poisons using a safety pipette, burette or measuring cylinder; mouth pipetting should be totally forbidden.

\section{Organic Solvents}

Which form a commonly used group of substances may have toxic properties. Thus benzene is toxic to bone marrow, carbon tetrachloride and other halogenated hydrocarbons to the liver.

\section{Mercury}

Lose vapour into the atmosphere if exposed at ordinary temperatures. If ventilation is poor and appreciable quantities are left exposed, dangerous amount can be inhaled.

\section{Toxic Gases}

Most likely to be encountered are natural gas, carbon dioxide, phosgene and compressed air. The risk of carbon monoxide 
poisoning with natural gas is slight, unless this is burning under very abnormal conditions where the air supply is restricted. Carbondioxide is only likely to reach hazardous concentrations where the solid form, dry ice is stored in a poorly ventilated room. Phosgene (carbonyl chloride) may be formed in stored chloroform residues and may be liberated during distillation

\section{Carcinogens}

Many chemicals have the potential to cause cancer. As the effects of carcinogens are irreversible, these substances should only be used if the work requiring them is essential and no alternative reagent is available. Those most commonly used in clinical biochemistry were aromatic amines such as benzidine and ortho-tolodine.

\section{Explosive and Flammable Chemicals}

Explosions may be produced when using certain chemicals, mainly oxidizing agents. Especially important is perchloric acid used to digest organic matter. As picric acid when dry explodes on percussion, it should not be kept in ground glass stopper. Kept in ground glass stopper bottles, but stored under water in a container closed by cork or rubber stopper. Ammoniacal silver nitrate should always be prepared freshly for use. Sodium azide used as a bacteriostatic agent, may produce metallic azides by reacting with metal waste pipes during disposal. The mixture of solvent vapour and air may be flammable. The gases include hydrogen, propane and acetylene. Oxygen is an additional hazard if used with any flammable chemical.

Again the wide range of concentration of flammable mixture with air or oxygen and the ease of ignition should be remembered. Keep cylinders not in use whether full or apparently empty, outside the laboratory in a special store in racks. Never use grease or oil to lubricate the threads of reducing valves. They are likely to cause explosion when used with oxygen cylinders. Cylinders are patients in various colour codes according to contents and gases for industrial use may have a different colour code from those for 'medical' use. If flammable gases are used with compressed air or oxygen, fit the gas line with a non-return valve. Taps for natural gas should be fitted towards the back of the bench to avoid turning on accidentally.

\section{Outbreaks of Fire}

If a fire breaks out, prompt, correct action will often limit the damage. The first action must be to raise the alarm. The fire alarm should be designed to alert the hospital switchboard automatically and ensure that the fire brigade is called.

\section{Time of Collection of Blood Specimens}

Some blood constituents such as iron and cortisol show a diurnal variation with highest concentrations during the morning, so the best time of collection will be determined by this.

\section{Avoiding Haemolysis}

Haemolysis is minimized by avoiding mechanical breakdown of red cells and movement of water out of or into the cells. When taking blood, constrict the arm minimally, draw the blood slowly and steadily into the syringe and expel it slowly and gently into the container with syringe tip touching the side, so that the blood runs slowly down. Do not expel blood through the needle.

\section{Changes in Blood on Keeping}

\section{Several changes may take place}

1. Loss of carbondioxide. As the carbondioxide content of plasma exceeds that of air, it diffuses from the plasma to the atmosphere and from cells to the plasma. This loss makes the blood more alkaline. The $\mathrm{pH}$ change is lessened by the conversion of bicarbonate ions to carbondioxide and water using hydrogen ion derived from other blood buffer systems.

2. Conversion of glucose to lactic acid. This is glycolysis. Glucose disappears fairly rapidly from whole blood on standing, so that up to $0.5 \mathrm{mmol} / \mathrm{L}$ may be lost hourly at room temperature.

3. Formation of ammonia from nitrogenous substances, of which urea is the chief may occur quickly. It is increased if the blood has been contaminated with bacteria.

\section{Collection of Urine Specimens}

Single specimens of urine are used for ward examinations and qualitative tests, but for quantitative work $24 \mathrm{hrs}$ collections are preferable.

\section{Changes on Storage}

The chief changes on storage arise from bacterial action. Urine for chemical examination is not usually collected aseptically and easily becomes infected. The most important effect of bacteria is to convert urea into ammonium carbonate accounting for the ammoniacal odour and alkalinity of grossly contaminated urine.

A high protein diet increases the volume because of the diuretic effect of the increased amount of urea formed. If the ambient temperature is high, volume is diminished and exercise has a similar effect.?

\section{REFERENCES}

1. http/www.sjsu/faculty/chem55/55gl part.htm

2. World Health Organization on behalf of the special program for Research and Training in Tropical diseases 2009.

3. Shirlyn B McKenzie. Clinical laboratory hematology, quality assessment in hematology, Second edition Vol 2, Pearson Education Inc, 1 Lake Street, New Jersey 07458, Page 925-939, 2010.

4. Culling CFA. Handbook of Histopatholgical Technique, Fixation, Processing, Section cutting, IInd Edition, Butter work \& co ltd, pages 25-97 \& 141-154, 1913.

5. Zuber M Naib. Text Book of Cytopathology, New Technique, $4^{\text {th }}$ Edition, Brown \& co, Pages 1-7 \& 125-130, 1985.

6. http://d.umn.edu/ rhicks/genmicro/Microbiology $\% 2$ 0Lab\%20Safety.pdf.

7. Alan H Gowenlock. Varley's practical clinical biochemistry, Hazards in the clinical biochemistry laboratory, collection of specimens, $1^{\text {st }}$ Edition, CBS publisher \& Distributors New Delhi, 2001, page - 1-21 \& 312-332. 